\title{
Constrained Source Space MR Spectroscopy: Multiple Voxels, No Gradient Readout
}

\author{
K. Landheer, A. Sahgal, S. Das, and S.J. Graham
}

\begin{abstract}
BACKGROUND AND PURPOSE: Our goal was to develop a novel technique for measuring a small number of localized spectra simultaneously and in a time-efficient manner.

MATERIALS AND METHODS: Using appropriate radiofrequency pulses, the magnetization from multiple voxels is excited simultaneously and then separated (reconstructed) by using the individual coil-sensitivity profiles from a multichannel receiver coil. Because no gradients are used for $k$-space encoding, constrained source space MR spectroscopy provides a time advantage over conventional spectroscopic imaging and an improved signal-to-noise ratio per square root of unit time over single-voxel spectroscopy applied at each successive location. In the present work, we considered prototype application of constrained source space MR spectroscopy for 2 voxels.
\end{abstract}

RESULTS: Experimental data from healthy volunteers and simulation results showed that constrained source space MR spectroscopy is effective at extracting 2 independent spectra even in the challenging scenario of the voxels being closely spaced. Also, from 6 patients with various types of brain cancer we obtained 2-voxel constrained source space MR spectroscopy data, which showed spectra of clinical quality in half the time required to perform successive single-voxel MR spectroscopy.

CONCLUSIONS: Constrained source space MR spectroscopy provides clinical quality spectra and could be used to probe multiple voxels simultaneously in combination with Hadamard encoding for further scan-time reductions.

ABBREVIATIONS: CSSMRS = constrained source space MR spectroscopy; PRESS = point-resolved spectroscopy sequence; SVS = single-voxel spectroscopy

$\mathbf{T}$

here are 2 major categories of MR spectroscopy pulse sequences on current clinical MR imaging systems: single-voxel spectroscopy (SVS), which measures one voxel, and MR spectroscopic imaging, which measures many spectra simultaneously over a Cartesian grid of voxels. Both SVS and MR spectroscopic imaging are widely applied in humans to detect certain molecular constituents of normal and abnormal tissues, especially those associated with cellular metabolism, and to monitor therapeutic response. ${ }^{1-3}$ Each MR spectroscopy category has its application niche, because SVS and MR spectroscopic imaging exploit dif-

Received September 26, 2014; accepted after revision January 19, 2015.

From the Departments of Medical Biophysics (K.L., S.J.G.), Surgery (S.D.), and Radiation Oncology, Sunnybrook Odette Cancer Centre (A.S.), University of Toronto, Ontario, Canada; Physical Sciences (K.L., S.J.G.), Sunnybrook Research Institute, Toronto, Ontario, Canada; and Keenan Research Centre (S.D.), St. Michael's Hospital, Toronto, Ontario, Canada.

K. Landeer was supported by scholarships from the Natural Sciences Research Council of Canada, the Ontario Graduate Scholarship program, and the Walter C. Sumner foundation.

Please address correspondence to Karl Landheer, MSc, Sunnybrook Research Institute, 2075 Bayview Ave, Room S605, Toronto, Ontario M4N 3M5, Canada; e-mail: landheer@sri.utoronto.ca

http://dx.doi.org/10.3174/ajnr.A4319 ferent spatial and temporal resolution trade-offs. SVS is attractive when anatomic MR imaging provides a precise indication of where spectral information should be collected. When pathology is more diffuse, widely distributed, or not detectable on anatomic MR imaging, MR spectroscopic imaging is the technique of choice for generating spectra from many voxels by using multiple repetitions for $k$-space encoding. ${ }^{4,5}$ To reduce spectroscopic scan times, various "parallel imaging" approaches have been applied to reduce the amount of $k$-space data acquired. These techniques exploit the spatial sensitivity of individual elements in multichannel receiver coils ${ }^{6-9}$ and can substantially reduce scan times.

The spatial limitations of SVS are well recognized; it is usually the case that SVS spectra are required at more than one location, either to compare spectra from diseased and normal tissue or in the case of multifocal disease. This limitation naturally leads to execution of SVS pulse sequences successively for each voxel location. There have been some attempts to modify spectroscopy acquisition to extend the volume of SVS coverage, such as with line-scan echo-planar spectroscopic imaging, ${ }^{10}$ which provides spectra from a column of voxels. However, for clinical applica- 

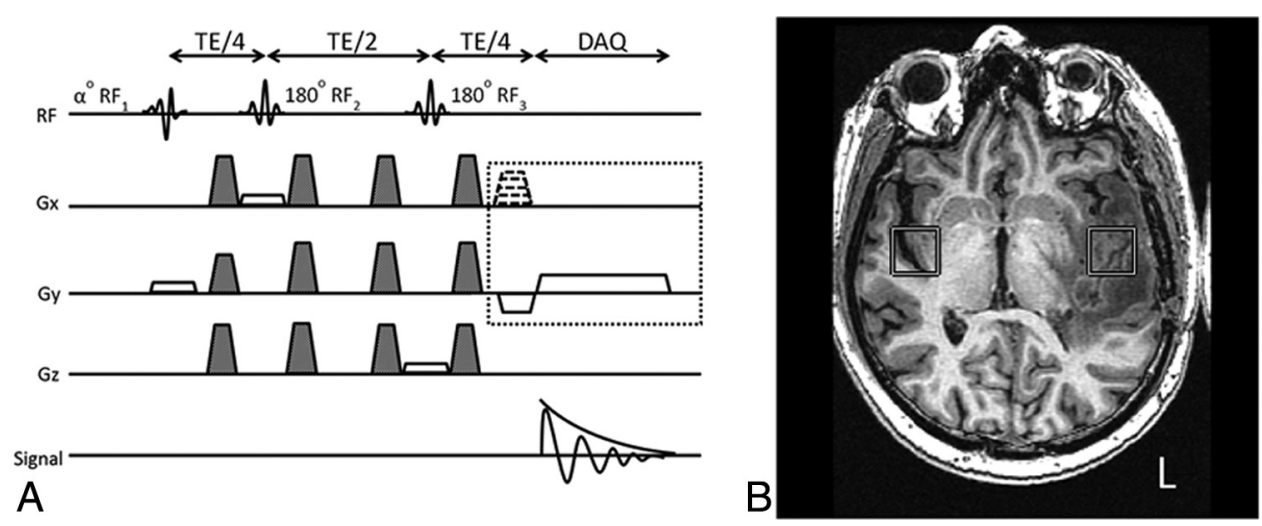

FIG 1. A, Pulse diagram for CSSMRS. The first radiofrequency pulse has a flip angle of $\alpha$ (where $\alpha$ is $<90^{\circ}$ ) and is cosine modulated, such that the subsequent spin echo after the third radiofrequency pulse excites 2 voxels. Shaded gradients are crusher gradients. The section-select rephasing lobe for the $y$ gradient is added directly to the first crusher. The gradient-echo readout in the dotted box is optional for voxel localization verification. RF indicates radiofrequency; DAQ, data acquisition. $B$, An anatomic Tl-weighted image of patient 6 with the nominal voxel locations overlaid and a brain tumor evident in the left middle temporal gyrus. The 2 spectra for this patient are displayed in the bottom row of Fig 2.

Table 1: Summary of patients with brain tumor studied in experiment 2

\begin{tabular}{|c|c|c|c|c|c|c|}
\hline $\begin{array}{l}\text { Patient } \\
\text { No. }\end{array}$ & $\begin{array}{l}\text { Age } \\
\text { (yr) }\end{array}$ & Sex & Disease & Radiation Treatment Status & Tumor Location & $\begin{array}{c}\text { Tumor Size } \\
\text { (vs Voxel Size) }\end{array}$ \\
\hline 1 & 36 & $\mathrm{~F}$ & Grade II oligodendroglioma & None & Right cingulate gyrus & Larger \\
\hline 2 & 84 & M & Grade IV glioblastoma & Currently undergoing focused radiation & Left middle temporal gyrus & Comparable \\
\hline 3 & 79 & M & Grade IV glioblastoma & Currently undergoing focused radiation & Left superior temporal gyrus & Larger \\
\hline 4 & 61 & $\mathrm{~F}$ & $\begin{array}{l}\text { Brain metastases from } \\
\text { breast cancer }\end{array}$ & $\begin{array}{l}60 \text { days since completion of focused } \\
\text { radiation }\end{array}$ & Left middle temporal gyrus & Smaller \\
\hline 5 & 79 & M & $\begin{array}{l}\text { Brain metastases from } \\
\text { colon cancer }\end{array}$ & $\begin{array}{l}70 \text { days since completion of focused } \\
\text { radiation }\end{array}$ & Right superior temporal gyrus & Smaller \\
\hline 6 & 61 & M & Grade IV glioblastoma & Currently undergoing focused radiation & Left middle temporal gyrus & Larger \\
\hline
\end{tabular}

Note:-F indicates female; $M$, male.

tions, standard SVS methods, notably point-resolved spectroscopy (PRESS) $)^{11}$ and stimulated echo acquisition mode, ${ }^{12}$ remain entrenched.

Previously, a technique that uses radiofrequency localization and sensitivity encoding ${ }^{13}$ was developed for fast functional MR imaging. ${ }^{14}$ It is reasonable that this "constrained source space" approach, appropriately modified for MR spectroscopy applications, should be investigated more to determine whether it usefully augments existing SVS capabilities. In the present work, in which we used constrained source space MR spectroscopy (CSSMRS), a prototype pulse sequence was developed and analyzed for its ability to acquire and separate spectra from 2 voxels simultaneously with no $k$-space encoding. The efficacy of spectral separation was investigated for a variety of distances between the 2 voxels in a healthy volunteer. In addition, numeric simulations were performed to assess the validity of certain assumptions made in the reconstruction and to predict CSSMRS performance in cases in which lengthy experimentation was impractical. Last, 2-voxel CSSMRS data were reported in relation to conventional SVS data acquired successively at each voxel location for patients with a variety of different brain cancers ranging from low grade to high grade.

\section{MATERIALS AND METHODS \\ CSSMRS Implementation and Spectral Analysis}

All experimental data were collected by using a Discovery MR750 3T imaging system (GE Healthcare, Milwaukee, Wisconsin) with a standard 8-channel head coil receiver. To achieve CSSMRS for proof-of-principle demonstrations, a standard PRESS sequence was modified to excite 2 voxels (instead of 1 ) arbitrarily in space (Fig 1A). Illustrative voxel locations are shown overlaid on the anatomic image of a patient with brain cancer in Fig $1 B$ (see patient 6 data in Table 1). The 2 user inputs were the voxel size, chosen throughout as $20 \mathrm{~mm} \times 20 \mathrm{~mm} \times 20 \mathrm{~mm}$, and the $\mathrm{x}, \mathrm{y}$, and $\mathrm{z}$ coordinates of each voxel location. In this approach, 2 arbitrarily positioned voxels were excited via cosine modulation of the first radiofrequency pulse, which resulted in the excitation of 2 parallel sections, followed by the standard spin-echo formation process thereafter. Arbitrary localization was obtained by modifying the offset frequencies of the radiofrequency pulses and changing the rotation array between logical and physical gradients. The 3 radiofrequency pulses were Shinnar-Le Roux pulses ${ }^{15}$ with durations of 3600, 5200, and 5200 milliseconds and bandwidths of 2366.67, 1384.62, and $1384.62 \mathrm{~Hz}$ for the first, second, and third pulses, respectively.

The additional pulse sequence parameters for this initial work included a TR and TE of 1500 milliseconds and 288 milliseconds (unless otherwise stated), respectively, a flip angle of $63^{\circ}$ (approximately the Ernst angle), a readout bandwidth of $2500 \mathrm{~Hz}$, and 1024 points of data acquisition (total acquisition time, 409.6 milliseconds). A TE value of 288 milliseconds was chosen because it has been shown to have high MR spectroscopy reproducibility, ${ }^{16}$ an important clinical factor compared with the other common TE values of 30 and 144 milliseconds, despite the associated reduction in SNR. Water suppression was implemented by us- 
ing chemical shift selective saturation. ${ }^{17}$ Before each data acquisition, first- and second-order shimming was applied to encompass most of the brain to decrease spectral linewidths. The typical linewidth of the water peak was approximately 8 Hz. The total number of excitations was 128 , and the total scan time was 3.2 minutes.

Regarding spatial reconstruction of CSSMRS data to separate spectra from the 2 voxels, the governing equation can be expressed in matrix form as follows ${ }^{13,14}$ :

$$
y(t)=S x(t)+\epsilon(t)
$$

where the sensitivity matrix $S$ relates how the magnetization signals $x(t)$ from each voxel result in the acquired signals $y(t)$ from each element in the receiver coil, and $\epsilon(t)$ represents coil element-dependent noise. As can be seen from Equation 1, the reconstruction of CSSMRS requires a calibration scan for the measurement of the coil sensitivity. The sensitivity matrix is generated by assuming that the spatial sensitivity $C$ of each coil element varies slowly over the extent of each voxel. For each of the $l$ coils and $n$ sections,

$$
S_{l, n}=\frac{1}{N_{p}} \sum_{i=i_{\min }}^{i_{\max }} \sum_{j=j_{\min }}^{j_{\max }} C_{l, n}(i, j),
$$

where $N_{p}$ is the number of pixels within the specified region inside the voxel, $i_{\min }$ and $i_{\max }$ are the minimum and maximum, respectively, row pixel limits on the voxel, and $j_{\min }$ and $j_{\max }$ are the minimum and maximum, respectively, column pixel limits on the voxel. Equation 1 can be solved by the sensitivity encoding formalism by using weak reconstruction with SNR optimization ${ }^{13}$ :

$$
\hat{x}\left(T R_{p} ; t\right)=\left(S^{H} \Psi^{-1} S\right)^{-1} S^{H} \Psi^{-1} y\left(T R_{p} ; t\right),
$$

where $\hat{x}$ is the estimated magnetization signal for each voxel, $T R_{p}$ denotes the $p$ th repetition, and $\Psi$ represents the noise covariance matrix between the coils. For example,

$$
\Psi_{l_{1}, l_{2}}=\frac{1}{N_{E}} \sum_{i=1}^{N_{E}}\left(\epsilon_{l_{1}}^{*}(i)-\overline{\epsilon_{l_{1}}^{*}}\right)\left(\epsilon_{l_{2}}(i)-\overline{\epsilon_{l_{2}}}\right),
$$

where the complex noise samples can be taken from the last datum of each acquisition, and the covariance is calculated over the total number of excitations, $N_{E}$.

The $C$ matrices can be estimated by various approaches, ${ }^{18-20}$ though previous CSS work has shown that a simple procedure is sufficient for proof-of-concept implementation. ${ }^{14}$ Two sets of fast gradient-echo images were acquired with the same pulse sequence parameters (TE, 1.3 milliseconds; TR, 34 milliseconds; flip angle, $5^{\circ}$; field of view, $30 \mathrm{~cm} ; 64 \times 64$ acquisition matrix; section thickness, $5 \mathrm{~mm}$ ): 1 set with the body coil and 1 set with the multichannel head coil receiver. These images were then interpolated to produce $256 \times 256$ images with an isotropic in-plane resolution of $1.17 \mathrm{~mm}$. For each of the $l$ coils and $n$ sections, the coil-sensitivity map at each in-plane $x, y$ coordinate, $C_{l, n}(x, y)$, was estimated by dividing each of the individual head coil images by the analogous body coil image and then thresholding by using an "object indicator" to set the coil sensitivity to 0 in regions in which noise dominated the object signal.

The CSSMRS reconstruction was performed by using specially written scripts in Matlab (MathWorks, Natick, Massachusetts).
The 2 separated signals were first zero-filled by a factor of 2 and then transformed to the spectral domain by fast Fourier transformation. The spectra were then phase corrected including zeroand first-order correction terms by using an automated algorithm based on minimizing entropy. ${ }^{21}$ The spectra were then shifted in frequency to place the peak for NAA at $2.04 \mathrm{ppm}$, normalized by their L2 norm, and subjected to Hankel-Lanczos singular value decomposition $^{22}$ for the removal of residual spectral content arising from water. Spectral components were then quantified automatically by using the freeware SPID (http://homes.esat. kuleuven.be/ biomed/software.php), which uses a separable nonlinear least-squares fitting algorithm known as automated quantitation of the short echo time MR spectroscopy spectra. ${ }^{22}$ The automated quantitation of short echo time MR spectroscopy spectra algorithm provides Cramer-Rao lower-bound estimates of the standard deviation of each quantified spectral component. The basis set used was simulated by using Java Magnetic Resonance User Interface (jMRUI) and the scan parameters. The values obtained from the quantification algorithm for NAA, Cho, and Cr were then scaled by attenuation factors to account for transverse and longitudinal relaxation effects by using relaxation constants obtained in a normal brain. ${ }^{23}$ According to common practice, the values for lactate were not adjusted for attenuation.

\section{Experimental Validation}

Bloch equation simulations confirmed that cosine modulation had negligible effects on the integrity of the spatial profile. A water-fat phantom was used to measure the signal bleed between voxels. One voxel was placed inside a stationary fat container, and another voxel was placed inside a surrounding water bath. Typical scan parameters, except a TE of 30 milliseconds for increased SNR, were used. This scan was repeated for center-to-center distances of 30-70 $\mathrm{mm}$. The bleed was defined to be the amplitude of the contaminating spectrum divided by the amplitude of the main spectrum in the other voxel multiplied by $100 \%$. Two validation experiments were subsequently conducted on healthy volunteers and patients with brain cancer to assess CSSMRS capabilities in practical scenarios. Each volunteer participated with free and informed consent and with the approval of the hospital research ethics board.

Experiment 1 was performed to investigate how CSSMRS results are affected by voxel placement in relation to coil-sensitivity profiles. Because CSSMRS involves sensitivity encoding reconstruction, overall performance depends on the condition number of the reconstruction matrix, as quantified by the $\mathrm{g}$ factor ${ }^{13}$ :

$$
g(k)=\sqrt{\left(S^{\star} \Psi^{-1} S\right)_{k, k}^{-1}\left(S^{\star} \Psi^{-1} S\right)_{k, k}},
$$

where the integer $k$ is used to denote the different voxels that are reconstructed (ie, $k=[1,2]$ in this case). To assess CSSMRS results for various $g$ factors, one voxel was placed in a fixed central location in the brain, and the other was placed to achieve center-to-center separations between voxels varying from $20 \mathrm{~mm}$ (ie, adjacent voxels) to $70 \mathrm{~mm}$ in the radial direction toward the head coil. SVS PRESS data were acquired in each successive location for comparison. These CSSMRS and PRESS data were collected for one healthy adult male (23 years old). Equation 2 was then used to calculate the sensitivity ma- 

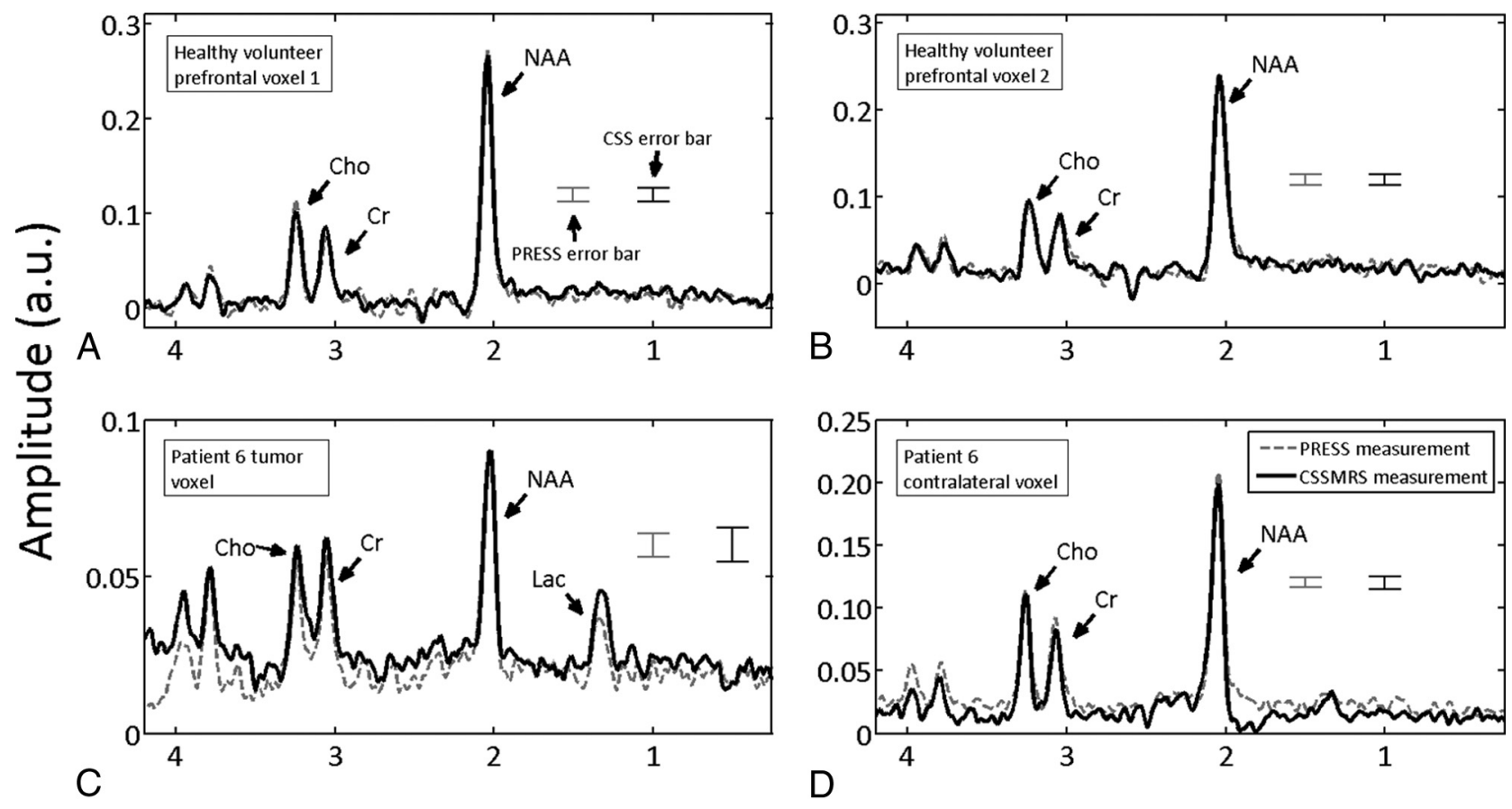

\section{Chemical shift (ppm)}

FIG 2. Spectra from a healthy volunteer $(A$ and $B)$ and a patient with brain cancer ( $C$ and $D)$ measured with both CSSMRS and PRESS. Spectra from patient 6 are shown, because this patient exhibited the median g-factor, typifying CSSMRS reconstruction quality. Errors represent the standard deviation over 128 excitations. a.u. indicates arbitrary units.

trix from the measured coil sensitivities at each individual voxel location, which, along with the noise covariance matrix (Equation 4), can be used to calculate the $\mathrm{g}$ factor by using Equation 5. In addition to these experiments, a single scan was performed on a healthy volunteer with a TE of 30 milliseconds to investigate the short echo time regimen.

Experiment 2 was performed to investigate how well CSSMRS distinguishes spectra from cancerous and normal tissue over a representative range of clinical presentations. Six patients with brain cancer were recruited from the Sunnybrook Odette Cancer Centre during the course of their treatment (see Table 1 for tumor characteristics). Patients were included if they presented with a tumor volume approximately the same size as the prescribed voxel, or slightly larger or smaller. Tumor location was verified by using a high-resolution fast-spoiled gradient-echo with an anatomic inversion recovery preparation (acquisition parameters are shown below). For each patient, one voxel was placed at the center of the tumor, and the other was placed on the contralateral side in the analogous neuroanatomic region within normal-appearing brain tissue. PRESS data were also acquired successively in these 2 locations for comparison purposes.

In both experiments, PRESS was performed with the identical acquisition parameters used in CSSMRS and with the same spectral analysis pipeline. The total examination time for comparing CSSMRS and PRESS data from 2 voxels was approximately 20 min, which included scout images, anatomic MR imaging (fastspoiled gradient-echo with an anatomic inversion recovery; $256 \times 256$ pixels; pixel size, $0.86 \times 0.86 \mathrm{~mm}$; TR, 8.2 milliseconds; TE, 3.2 milliseconds; flip angle, $8^{\circ}$ ), and 2 fast gradient-echo scans (for measuring coil sensitivity, as already described) and highorder shim, CSSMRS, and PRESS acquisitions.

\section{Numeric Simulation}

A simple numeric simulation was also written in Matlab for additional insight into the results of experiments 1 and 2. The simulation assessed the impact on spatial reconstruction of the important assumption underlying Equation 1, namely that coil sensitivity could be reasonably approximated as a constant over each voxel. Given good agreement between experimental results and simulations for experiment 1 (see "Results"), the simulation also was used to predict CSSMRS performance under conditions that were not possible to measure experimentally during experiment 2 because of the inherent time restrictions for collecting MR spectroscopy data in patients.

The simulation used measured coil-sensitivity and PRESS data from 2 voxels as initial inputs. In the context of the simulation, the PRESS data (obtained according to the experimental parameters already given, averaged over 128 excitations) were considered to represent a situation in which signal components were concentrated uniformly over each voxel volume. Simulated signals were then generated for each coil element, while accounting for nonuniform coil sensitivity, by performing the appropriate spatial integral. Complex Gaussian noise was added to each simulated signal to approximate the levels observed experimentally for each coil. These simulated coil signals were then used for spatial reconstruction of 2 voxel signals according to Equations 1-4 for subsequent comparison with the PRESS data that were input originally. Spatial recon- 


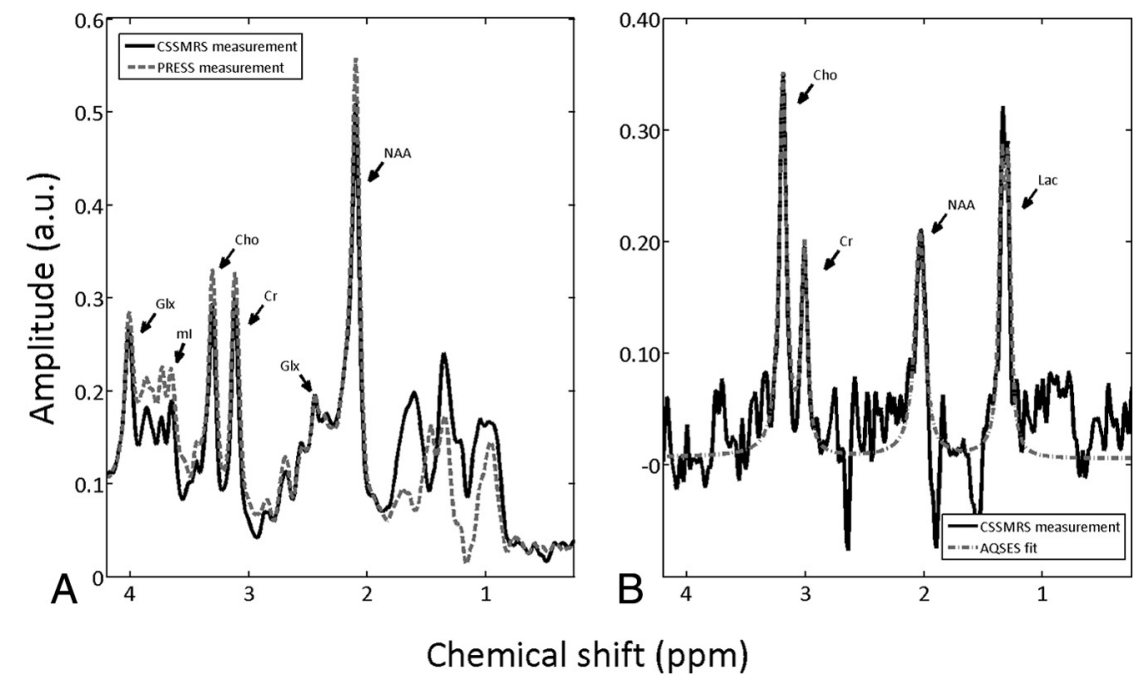

FIG 3. A, Spectra from a healthy volunteer at 30-millisecond echo time, obtained by using both CSSMRS and PRESS. The labeled metabolites are myo-inositol (ml), Cho, Cr, Glx, and NAA. B, The unapodized spectrum obtained from CSSMRS from patient 1 (highest g-factor) along with the automated quantitation of short echo time MR spectroscopy spectra (AQSES) fit.

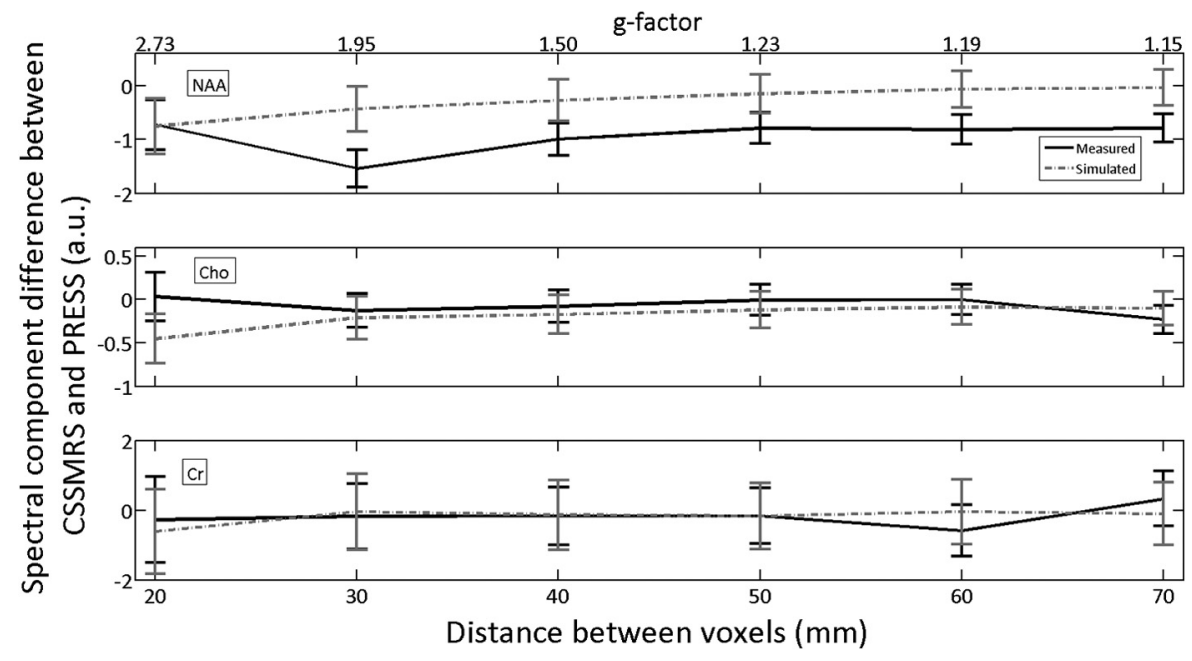

FIG 4. Measured and simulated differences between the CSSMRS and PRESS measurement for 6 different voxel separations for the 3 main metabolites within a healthy adult brain: NAA, Cho, and Cr. The signal from the CSSMRS voxel that was kept in a fixed position was reconstructed and compared with the PRESS measurement obtained from the same location. The black and gray lines represent the measured and simulated values, respectively. The $g$ factors are also displayed for reference above the top $\mathrm{x}$-axis, though there is a nonlinear relationship between $\mathrm{g}$ factor and voxel separation. Error bars represent Cramer-Rao bounds.

struction, spectral processing, and analysis were conducted as outlined above for experimental data.

\section{RESULTS}

For center-to-center spacings of 30, 40, 50, 60, and $70 \mathrm{~mm}$, the observed bleeds of water into the fat voxel were $6.2 \%, 6.3 \%, 3.5 \%, 0.4 \%$, and $1.7 \%$, respectively, and the observed bleeds of fat into the water voxel were $3.0 \%, 0.2 \%, 0.1 \%, 5.2 \%$, and $2.4 \%$, respectively.

For visual comparison, Fig 2 displays 4 representative spectra obtained by CSSMRS (solid black lines) and PRESS (dashed gray lines). As commonly performed for display purposes, all spectra were apodized by a Gaussian filter with $2 \mathrm{~Hz}$ full-width at halfmaximum. The spectra shown in Fig $2 A,-B$ are qualitatively similar and were obtained from a healthy volunteer with both voxels placed inside the prefrontal cortex. The spectra shown in Fig 2C, $-D$ were obtained from patient 6 (Table 1) and are substantially different for the 2 voxels, with the spectra in Fig $2 C$ obtained from tumor tissue inside the left middle temporal gyrus and those in Fig $2 D$ obtained from contralateral homologous tissue, as shown in Fig $1 B$. Spectra from patient 6 were chosen for display in Fig 2 because CSSMRS results were obtained in this case with the median $g$ factor observed over the patient cohort. Figure $3 A$ displays spectra obtained from both CSSMRS and PRESS for the minimum achievable TE of this pulse sequence (30 milliseconds). Figure $3 B$ shows the tumor spectrum obtained from CSSMRS for patient 1 and the fit obtained from automated quantitation of short echo time MR spectroscopy spectra.

The results of experiment 1 and related numeric simulations are shown in Fig 4, in which are plotted the difference between quantified spectral components measured by CSSMRS and PRESS for 6 different voxel separations (one voxel held fixed, one moved radially) and the 3 main metabolites observed in Fig $2 A$, $-B$ : NAA, Cr, and Cho. The difference values (CSSMRS minus PRESS) reported are specifically for the voxel that was maintained in a fixed position. For both the experimental and simulated results, the difference between CSSMRS and PRESS remained constant within experimental error over all the voxel separations. Furthermore, the difference values for experimental and simulation results also agreed within error, with the only exception being a slight bias in NAA quantification when voxels were separated by more than $20 \mathrm{~mm}$.

Given the good level of agreement between experiment and simulation observed in Fig 4, numeric simulations were then extended to assess CSSMRS reconstruction quality as a function of voxel separation with spectra that were substantially different in the 2 voxels. Figure 5 shows plots of the difference between quantified spectral components measured by CSSMRS and PRESS in a manner analogous to that shown in Fig 4; however, in this case, the inputs to the simulation were provided from patient 6 with the difference values relating to quantification of the tumor spectral components: NAA, Cho, Cr, and lactate. For additional 


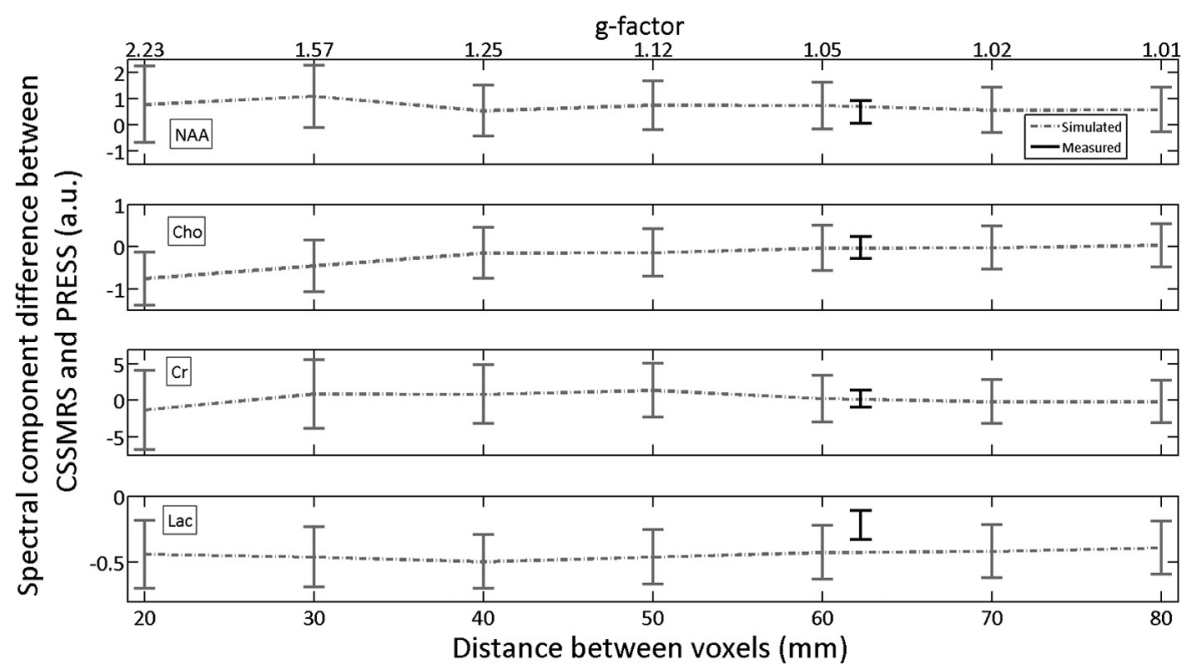

FIG 5. Simulated metabolite quantification values for 7 different voxel separations for the 4 main metabolites within the tumor spectra for patient 6: NAA, Cho, Cr, and lactate (Lac). The quantified values were from the stationary voxel placed within the tumor and are plotted in gray. The black data points located at $62 \mathrm{~cm}$ in each plot are the experimental results for this patient, corresponding to the first difference column values listed in Tables $2-5$ for patient 6 . The estimated $g$ factors are also displayed above the top $\mathrm{x}$-axis for reference, though there is a nonlinear relationship between the $g$ factor and voxel separation. context, the difference values obtained experimentally for patient 6 are also indicated as single data points in Fig 5. Similar to Fig 4, Fig 5 shows difference values of 0 within error for all voxel separations and metabolites (except Cho for adjacent voxels), indicating that good CSSMRS reconstruction quality was maintained even when the 2 voxels were located in close proximity to one another. In addition, the reconstruction tends to improve as the distance between the voxels increases for all metabolites. The simulation results and experimental results also agree within error for the single experimental data point.

To summarize the results of experiment 2, CSSMRS and PRESS results are quantified in Tables $2-5$ for patients 1-6 across tumor and normal tissue voxels for NAA, Cr, Cho,

Table 2: Quantified NAA values from PRESS and CSSMRS for both voxels ${ }^{\mathrm{a}}$

\begin{tabular}{lcccccr}
$\begin{array}{c}\text { Patient } \\
\text { No. }\end{array}$ & $\begin{array}{c}\text { CSSMRS Tumor } \\
\text { Voxel (a.u.) }\end{array}$ & $\begin{array}{c}\text { PRESS Tumor } \\
\text { Voxel (a.u.) }\end{array}$ & $\begin{array}{c}\text { Difference } \\
\text { (a.u.) }\end{array}$ & $\begin{array}{c}\text { CSSMRS Healthy } \\
\text { Voxel (a.u.) }\end{array}$ & $\begin{array}{c}\text { PRESS Healthy } \\
\text { Voxel (a.u.) }\end{array}$ & $\begin{array}{c}\text { Difference } \\
\text { (a.u.) }\end{array}$ \\
\hline 1 & $5.70 \pm 0.65$ & $4.86 \pm 0.58$ & $0.83 \pm 0.87$ & $9.73 \pm 0.28$ & $9.88 \pm 0.20$ & $-0.15 \pm 0.35$ \\
2 & $2.70 \pm 0.44$ & $2.08 \pm 0.40$ & $0.62 \pm 0.59$ & $9.34 \pm 0.35$ & $8.84 \pm 0.35$ & $0.50 \pm 0.49$ \\
3 & $1.70 \pm 0.19$ & $1.60 \pm 0.20$ & $0.10 \pm 0.28$ & $9.14 \pm 0.12$ & $9.42 \pm 0.14$ & $-0.28 \pm 0.18$ \\
4 & $3.33 \pm 0.54$ & $0.14 \pm 0.25$ & $3.19 \pm 0.59$ & $8.09 \pm 0.38$ & $8.89 \pm 0.36$ & $-0.81 \pm 0.52$ \\
5 & $5.27 \pm 0.29$ & $4.22 \pm 0.21$ & $1.05 \pm 0.35$ & $8.87 \pm 0.38$ & $10.93 \pm 0.55$ & $-2.06 \pm 0.66$ \\
6 & $5.89 \pm 0.27$ & $5.41 \pm 0.33$ & $0.48 \pm 0.43$ & $6.81 \pm 0.21$ & $6.98 \pm 0.20$ & $-0.17 \pm 0.29$ \\
\hline
\end{tabular}

Note:-a.u. indicates arbitrary units.

a Shown are means and standard deviations (Cramer-Rao bounds).

Table 3: Quantified Cho values from PRESS and CSSMRS for both voxels ${ }^{\mathrm{a}}$

\begin{tabular}{lllcccc}
\hline $\begin{array}{c}\text { Patient } \\
\text { No. }\end{array}$ & $\begin{array}{c}\text { CSSMRS Tumor } \\
\text { Voxel (a.u.) }\end{array}$ & $\begin{array}{c}\text { PRESS Tumor } \\
\text { Voxel (a.u.) }\end{array}$ & $\begin{array}{c}\text { Difference } \\
\text { (a.u.) }\end{array}$ & $\begin{array}{c}\text { CSSMRS Healthy } \\
\text { Voxel (a.u.) }\end{array}$ & $\begin{array}{c}\text { PRESS Healthy } \\
\text { Voxel (a.u.) }\end{array}$ & $\begin{array}{c}\text { Difference } \\
\text { (a.u.) }\end{array}$ \\
\hline 1 & $2.93 \pm 0.39$ & $2.89 \pm 0.24$ & $0.04 \pm 0.45$ & $1.72 \pm 0.18$ & $1.73 \pm 0.13$ & $-0.01 \pm 0.22$ \\
2 & $1.86 \pm 0.27$ & $1.67 \pm 0.17$ & $0.19 \pm 0.32$ & $1.89 \pm 0.20$ & $2.22 \pm 0.22$ & $-0.33 \pm 0.30$ \\
3 & $0.67 \pm 0.12$ & $0.96 \pm 0.17$ & $-0.28 \pm 0.21$ & $2.06 \pm 0.07$ & $2.31 \pm 0.08$ & $-0.25 \pm 0.11$ \\
4 & $0.97 \pm 0.93$ & $0.83 \pm 0.33$ & $0.15 \pm 0.99$ & $1.36 \pm 0.18$ & $2.05 \pm 0.21$ & $-0.69 \pm 0.75$ \\
5 & $1.66 \pm 0.12$ & $1.81 \pm 0.11$ & $-0.15 \pm 0.16$ & $1.56 \pm 0.26$ & $3.02 \pm 0.66$ & $-1.46 \pm 0.71$ \\
6 & $1.56 \pm 0.17$ & $1.59 \pm 0.20$ & $-0.02 \pm 0.26$ & $1.75 \pm 0.13$ & $1.80 \pm 0.12$ & $-0.05 \pm 0.17$ \\
\hline
\end{tabular}

Note:-a.u. indicates arbitrary units.

a Shown are means and standard deviations (Cramer-Rao bounds).

Table 4: Quantified Cr values from PRESS and CSSMRS for both voxels ${ }^{\mathrm{a}}$

\begin{tabular}{|c|c|c|c|c|c|c|}
\hline $\begin{array}{l}\text { Patient } \\
\text { No. }\end{array}$ & $\begin{array}{l}\text { CSSMRS Tumor } \\
\text { Voxel (a.u.) }\end{array}$ & $\begin{array}{l}\text { PRESS Tumor } \\
\text { Voxel (a.u.) }\end{array}$ & $\begin{array}{l}\text { Difference } \\
\text { (a.u.) }\end{array}$ & $\begin{array}{l}\text { CSSMRS Healthy } \\
\text { Voxel (a.u.) }\end{array}$ & $\begin{array}{l}\text { PRESS Healthy } \\
\text { Voxel (a.u.) }\end{array}$ & $\begin{array}{l}\text { Difference } \\
\text { (a.u.) }\end{array}$ \\
\hline 1 & $10.62 \pm 2.44$ & $7.77 \pm 1.07$ & $2.85 \pm 2.66$ & $6.34 \pm 0.77$ & $6.50 \pm 0.61$ & $-0.16 \pm 0.98$ \\
\hline 2 & $3.82 \pm 1.68$ & $1.66 \pm 0.55$ & $2.16 \pm 1.77$ & $9.09 \pm 0.89$ & $10.40 \pm 1.07$ & $-1.32 \pm 1.39$ \\
\hline 3 & $2.41 \pm 0.77$ & $1.56 \pm 0.67$ & $0.85 \pm 1.02$ & $8.73 \pm 0.32$ & $8.37 \pm 0.35$ & $0.36 \pm 0.48$ \\
\hline 4 & $4.66 \pm 4.17$ & $0.42 \pm 0.63$ & $4.24 \pm 4.22$ & $8.94 \pm 1.03$ & $8.60 \pm 0.95$ & $-0.25 \pm 1.40$ \\
\hline 5 & $6.19 \pm 0.61$ & $5.98 \pm 0.48$ & $0.21 \pm 0.77$ & $10.44 \pm 1.76$ & $10.58 \pm 2.97$ & $-0.15 \pm 3.45$ \\
\hline 6 & $8.21 \pm 0.74$ & $8.00 \pm 0.88$ & $0.22 \pm 1.15$ & $6.13 \pm 0.56$ & $6.19 \pm 0.52$ & $-0.05 \pm 0.77$ \\
\hline
\end{tabular}

Note:-a.u. indicates arbitrary units

a Shown are means and standard deviations (Cramer-Rao bounds). 
Table 5: Quantified lactate values from PRESS and CSSMRS for both voxels ${ }^{\mathrm{a}}$

\begin{tabular}{lllcccc}
\hline $\begin{array}{c}\text { Patient } \\
\text { No. }\end{array}$ & $\begin{array}{c}\text { CSSMRS Tumor } \\
\text { Voxel (a.u.) }\end{array}$ & $\begin{array}{c}\text { PRESS Tumor } \\
\text { Voxel (a.u.) }\end{array}$ & $\begin{array}{c}\text { Difference } \\
\text { (a.u.) }\end{array}$ & $\begin{array}{c}\text { CSSMRS Healthy } \\
\text { Voxel (a.u.) }\end{array}$ & $\begin{array}{c}\text { PRESS Healthy } \\
\text { Voxel (a.u.) }\end{array}$ & $\begin{array}{c}\text { Difference } \\
\text { (a.u.) }\end{array}$ \\
\hline 1 & $1.31 \pm 0.09$ & $1.46 \pm 0.08$ & $-0.16 \pm 0.12$ & $0.03 \pm 0.04$ & $0.23 \pm 0.07$ & $-0.20 \pm 0.08$ \\
2 & $3.36 \pm 0.11$ & $3.16 \pm 0.10$ & $0.20 \pm 0.15$ & $0.60 \pm 0.11$ & $0.51 \pm 0.11$ & $0.09 \pm 0.16$ \\
3 & $2.83 \pm 0.04$ & $2.77 \pm 0.04$ & $0.06 \pm 0.05$ & $0.25 \pm 0.04$ & $0.28 \pm 0.05$ & $0.03 \pm 0.06$ \\
$4^{b}$ & $3.22 \pm 0.14$ & $2.76 \pm 0.13$ & $0.46 \pm 0.19$ & $1.84 \pm 0.10$ & $1.59 \pm 0.10$ & $0.25 \pm 0.14$ \\
5 & $2.26 \pm 0.08$ & $2.32 \pm 0.07$ & $-0.06 \pm 0.10$ & $0.73 \pm 0.13$ & $0.87 \pm 0.17$ & $-0.14 \pm 0.21$ \\
6 & $0.54 \pm 0.07$ & $0.76 \pm 0.08$ & $-0.22 \pm 0.11$ & $0.22 \pm 0.05$ & $0.29 \pm 0.05$ & $-0.08 \pm 0.07$ \\
\hline
\end{tabular}

Note:- a.u. indicates arbitrary units.

a Shown are means and standard deviations (Cramer-Rao bounds).

b Lipid contamination from scalp mislabeled as lactate in healthy voxel.

and lactate, including the differences in spectral quantification. The CSSMRS g factors for patients $1-6$ were 1.39, 1.00, 1.00, 1.13, 1.17 , and 1.01, respectively, which indicates that there should be a SNR per square root of unit time benefit for CSSMRS over PRESS in all cases. Overall, large decreases in NAA and increases in lactate and Cho were observed for tumor voxels in relation to normal tissue voxels for CSSMRS and PRESS for most patients, consistent with results from previous studies. ${ }^{24}$ Tables $2-5$ also show large variability in the tumor spectra across patients. A Mann-Whitney $U$ test on the pooled values from all metabolites obtained from CSSMRS versus PRESS yielded a $P$ value of .86 , indicating no significant difference. There was no evidence of significant voxel bleed in the in vivo experiments, because no systematic increase in lactate was observed in normal tissue CSSMRS voxels (Table 5), except that a large lactate value was obtained from CSSMRS and PRESS spectra in the healthy tissue of patient 4. Voxel placement was close to the scalp in this particular patient, which produced contaminating lipid signals that were subsequently misinterpreted as lactate by the automated quantitation of short echo time MR spectroscopy spectra software. Thus, this specific result should be discounted. In addition there was a significant increase observed for this patient in NAA in the CSSMRS tumor voxel, which is likely because of motion that exacerbated the bleed effects (this particular patient had difficulty remaining still).

\section{DISCUSSION}

This work has introduced a prototype pulse sequence for CSSMRS, a novel spectroscopy technique that measures spectra from multiple voxels simultaneously without the need for $k$-space encoding. Instead, spatial encoding is achieved by multivoxel radiofrequency selective excitation, signal readouts from a multichannel receiver coil, and sensitivity encoding ${ }^{14}$ reconstruction to separate the signals from each voxel. The CSSMRS method is important from the perspective of SNR per square root of acquisition time, potentially providing efficiency in comparison to the standard clinical practice of performing successive SVS acquisitions at different voxel locations.

Careful experiments and simulations were undertaken to investigate the capabilities of CSSMRS for simultaneous measurement of 2 voxels. In particular, considerable attention was paid to whether CSSMRS provides adequate spatial localization in relation to the standard SVS PRESS method. In a water-fat experiment, it was shown that the bleed rate was up to $6 \%$ for very closely spaced voxels and less for further spaced voxels. This amount of bleed is acceptable for spectroscopic applications. Ex- periments 1 and 2, conducted with healthy volunteers and a diverse group of 6 patients with a brain tumor (4 different types of cancer were represented), showed overall that CSSMRS and successive PRESS spectra agreed within experimental error. Furthermore, CSSMRS spatial reconstruction was shown to be robust over a range of voxel prescriptions (with one voxel held fixed and the voxel separation varied) by both experiments and numeric simulations. The experiment and simulation were in agreement for a healthy volunteer, indicating excellent reconstruction even when the 2 voxels were placed adjacent to one another. The only additional feature of note in this regard was a systematic decrease in the measured value of NAA in CSS (as shown in Fig 4), which was not predicted by simulation. This feature is likely a result of either motion or the relatively simplistic nature of the simulations, which did not account for various experimental factors. However, given that the overall level of agreement between experiment and simulation was very good, these factors evidently have a small influence. The simulation, therefore, helped to support the assumption made in CSSMRS reconstruction that coil-sensitivity variations can be neglected within the voxels.

The agreement between these experiments and the simulation provides rationale for using simulations to further predict CSSMRS capabilities in a patient with brain cancer. As expected, slightly larger variations were observed as a function of voxel separation in this case, likely because of the larger spectral differences between the 2 voxels. However, with the exception of NAA for voxels separated by greater than $20 \mathrm{~mm}$, all CSSMRS results were predicted to be consistent with PRESS results within error.

Given that CCSMRS has been demonstrated to provide robust high-quality results, discussion can turn productively to the potential efficiency of this pulse sequence in terms of SNR per square root of acquisition time. In the 2-voxel implementation investigated in the present work, spectra were obtained in half the time of successive application of PRESS. The quality of the CSSMRS results may have been affected by noise amplification in the sensitivity encoding reconstruction, however, as parameterized by the $\mathrm{g}$ factor. Therefore, the appropriate context for by using CSSMRS advantageously over PRESS is when the g factor is less than $\sqrt{ } 2$, which corresponds to a minimum center-to-center separation in voxels of approximately $35 \mathrm{~mm}$ near the center of the 8-channel head receiver coil used in this work. All patients had a $\mathrm{g}$ factor below this threshold.

CSSMRS performed similarly with a short echo time, though a notable increase in the lipid peaks was observed. This increase is likely a result of the slight modification of the pulse profile and 
will be corrected for in the future by using outer-volume suppression.

It is also interesting to note that CSSMRS is compatible with another approach that avoids using $k$ space for spatially encoding spectral information. In principle, if the flip angles assigned to each voxel can be modulated appropriately, then simple algebraic combinations of the successive spectroscopic readouts can be used to localize each voxel without sensitivity encoding reconstruction, as achieved in Hadamard spectroscopic imaging. ${ }^{24}$ The Hadamard spectroscopic imaging approach is independent of $\mathrm{g}$ factor and also provides improvements in SNR per square root of time but traditionally has required excellent radiofrequency fidelity and is sensitive to how spatial radiofrequency nonuniformity and patient motion influence algebraic combination and the subsequent leakage of signals between voxels. In addition, the algebraic combination of multiple recordings reduces the minimum temporal resolution that is achievable with Hadamard spectroscopic imaging, whereas CSSMRS provides spectral separation in as little as a single TR value. CSSMRS and Hadamard spectroscopic imaging are not mutually exclusive, however, and it is possible that a robust hybrid technique can be developed in the future for further scan-time reductions.

Irrespective of developing such a hybrid technique, the method discussed here has potential applications in any in vivo spectroscopy experiment in which there are 2 regions of interest and the lengthy acquisition times of MR spectroscopic imaging are impractical. CSSMRS may also be beneficial in a research setting in which sophisticated 2D MR spectroscopy experiments have inherently long acquisition times, such as J-resolved MR spectroscopy. ${ }^{25}$ Another promising application of CSSMRS is in functional spectroscopy, in which real-time changes in metabolic information can be measured from multiple points within the brain simultaneously with high temporal resolution. Further development and applications of CSSMRS should be explored in the future.

\section{CONCLUSIONS}

CSSMRS has been developed to extract signals from 2 localized regions simultaneously and reliably. Utility was demonstrated in a clinical setting, though the technique also has promising applications in research settings.

Disclosures: Karl Landheer-RELATED: Support for Travel to Meetings for the Study or Other Purposes: University of Toronto SGS Travel Award; UNRELATED: Grant: NSERC Canadian Graduate Scholarship. Sunit Das—RELATED: Grant: Canadian Cancer Society Research Institute.* *Money paid to institution.

\section{REFERENCES}

1. Frahm J, Bruhn H, Gyngell ML, et al. Localized proton NMR spectroscopy in different regions of the human brain in vivo: relaxation times and concentrations of cerebral metabolites. Magn Reson Med 1989;11:47-63

2. Spielman D, Pauly J, Macovski A, et al. Spectroscopic imaging with multidimensional pulses for excitation: SIMPLE. Magn Reson Med 1991;19:67-84

3. Segebarth CM, Balériaux DF, Luyten PR, et al. Detection of metabolic heterogeneity of human intracranial tumors in vivo by ${ }^{1} \mathrm{H}$ NMR spectroscopic imaging. Magn Reson Med 1990;13:62-76

4. Brown TR, Kincaid BM, Ugurbil K. NMR chemical shift imaging in three dimensions. Proc Natl Acad Sci U S A 1982;79:3523-26

5. Maudsley AA, Hilal SK, Perman WH, et al. Spatially resolved high resolution spectroscopy by "four-dimensional" NMR. J Magn Reson 1983;51:147-52

6. Lin FH, Tsai SY, Otazo R, et al. Sensitivity-encoded (SENSE) proton echo-planar spectroscopic imaging (PEPSI) in the human brain. Magn Reson Med 2007;57:249-57

7. Gu M, Liu C, Spielman DM. Parallel spectroscopic imaging reconstruction with arbitrary trajectories using $\mathrm{k}$-space sparse matrices. Magn Reson Med 2009;61:267-72

8. Posse S, Otazo R, Tsai SY, et al. Single-shot magnetic resonance spectroscopic imaging with partial parallel imaging. Magn Reson Med 2009;61:541-47

9. Bonekamp D, Smith MA, Zhu H, et al. Quantitative SENSE-MRSI of the human brain. Magn Reson Imaging 2010;28:305-13

10. Oshio K, Kyriakos W, Mulkern RV. Line scan echo planar spectroscopic imaging. Magn Reson Med 2000;44:521-24

11. Bottomley PA. Spatial localization in NMR spectroscopy in vivo. Ann NY Acad Sci 1987;508:333-48

12. Frahm J, Merboldt KD, Hänicke W. Localized proton spectroscopy using stimulated echoes. J Magn Reson 1987;72:502-08

13. Chiew M, Graham SJ. Constrained source space imaging: application to fast, region-based functional MRI. Magn Reson Med 2013;70:1058-69

14. Pruessmann KP, Weiger M, Scheidegger MB, et al. SENSE: sensitivity encoding for fast MRI. Magn Reson Med 1999;42:952-62

15. Pauly J, Le Roux P, Nishimura D, et al. Parameter relations for the Shinnar-Le Roux selective excitation pulse design algorithm [NMR imaging]. IEEE Trans Med Imaging 1991;10:53-65

16. Inglese M, Spindler M, Babb JS, et al. Field, coil, and echo-time influence on sensitivity and reproducibility of brain proton MR spectroscopy. AJNR Am J Neuroradiol 2006;27:684-88

17. Haase A, Frahm J, Hänicke W, et al. ${ }^{1} \mathrm{H}$ NMR chemical shift selective (CHESS) imaging. Phys Med Biol 1985;30:341-44

18. Jin J, Liu F, Weber E, et al. An electromagnetic reverse method of coil sensitivity mapping for parallel MRI-theoretical framework. $J$ Magn Reson 2010;207:59-68

19. Allison MJ, Ramani S, Fessler JA. Regularized MR coil sensitivity estimation using augmented Lagrangian methods. Proc IEEE Int Symp Biomed Imaging 2012;32:394-97

20. Allison MJ, Ramani S, Fessler JA. Accelerated regularized estimation of MR coil sensitivities using augmented Lagrangian methods. IEEE Trans Med Imaging 2013;32:556-64

21. Chen L, Weng Z, Goh L, et al. An efficient algorithm for automatic phase correction of NMR spectra based on entropy minimization. $J$ Magn Reson 2002;158:164-68

22. Poullet JB, Sima DM, Simonetti AW, et al. An automated quantitation of short echo time MRS spectra in an open source software environment: AQSES. NMR Biomed 2007;20:493-504

23. Li Y, Xu D, Ozturk-Isik E, et al. T1 and T2 metabolite relaxation times in normal brain at 3T and 7T. J Mol Imaging Dyn 2012;1:1-5

24. Horská A, Barker PB. Imaging of brain tumors: MR spectroscopy and metabolic imaging. Neuroimaging Clin N Am 2010;20:293-310

25. Ryner LN, Sorenson JA, Thomas MA. Localized 2D J-resolved ${ }^{1} \mathbf{H}$ MR spectroscopy: strong coupling effects in vitro and in vivo. Magn Reson Imaging 1995;13:853-69 